\title{
Resource acquisition, distribution and end-use efficiencies and the growth of industrial society
}

\author{
A. J. Jarvis ${ }^{1}$, S. J. Jarvis ${ }^{2}$, and C. N. Hewitt ${ }^{1}$ \\ ${ }^{1}$ Lancaster Environment Centre, Lancaster University, Lancaster, UK \\ ${ }^{2}$ Office of Gas and Electricity Markets, London, UK
}

Correspondence to: A. J. Jarvis (a.jarvis@lancs.ac.uk)

Received: 4 December 2014 - Published in Earth Syst. Dynam. Discuss.: 29 January 2015

Revised: 7 September 2015 - Accepted: 15 September 2015 - Published: 13 October 2015

\begin{abstract}
A key feature of the growth of industrial society is the acquisition of increasing quantities of resources from the environment and their distribution for end-use. With respect to energy, the growth of industrial society appears to have been near-exponential for the last 160 years. We provide evidence that indicates that the global distribution of resources that underpins this growth may be facilitated by the continual development and expansion of near-optimal directed networks (roads, railways, flight paths, pipelines, cables etc.). However, despite this continual striving for optimisation, the distribution efficiencies of these networks must decline over time as they expand due to path lengths becoming longer and more tortuous. Therefore, to maintain long-term exponential growth the physical limits placed on the distribution networks appear to be counteracted by innovations deployed elsewhere in the system, namely at the points of acquisition and end-use of resources. We postulate that the maintenance of the growth of industrial society, as measured by global energy use, at the observed rate of $\sim 2.4 \% \mathrm{yr}^{-1}$ stems from an implicit desire to optimise patterns of energy use over human working lifetimes.
\end{abstract}

\section{Introduction}

The growth of industrial society since the Industrial Revolution has required the continual exploitation of a diverse range of environmentally derived resources. Because resources are seldom consumed at the point of extraction, this in turn has required the construction of ever-expanding distribution networks. These networks can be seen to form part of a global Resource Acquisition, Distribution and End-use (RADE) system linking environmental resources with points of enduse. In many respects these man-made networks resemble those seen in natural systems, both in terms of form and function. Here we attempt to apply theoretical insights from research into the evolution of natural systems to the man-made system that constitutes global industrial society, with a particular focus on energy.

This paper builds on a long tradition of attempting to understand socio-economic systems through the application of insights from the natural sciences. Initially these insights were largely metaphoric, but increasingly the application of evolutionary (Nelson and Wilson, 1982), metabolic (Fischer-
Kowalski and Huttler, 1998) and thermodynamic (Garrett, 2011, 2012) theories has become much more direct in this area. The fundamental physical constraints that underpin the development of distribution networks have previously been used to try and explain the behaviour of biological systems (West et al., 1997), river basins (Rodríguez-Iturbe and Rinaldo, 1997), electricity grids, water distribution systems, road networks (Dalgaard and Strulik, 2011; Pauliuka et al., 2014; Bettencourt et al., 2007), and even cities (Bettencourt, 2013), but have not previously been applied to the behaviour and growth of global industrial society itself.

Here we explore the possibility that the growth of industrial society is in part regulated by the behaviour of the distribution networks within a global RADE system. The resources moved by man-made distribution networks include energy and the other materials from which industrial society is constructed. In the following analysis we focus specifically on the energy used in acquisition, distribution and enduse. We do this because the performance of RADE networks is determined by their energy efficiency (i.e. the proportion 
of energy lost in transporting mass across networks) and because energy use is one of the best observed metrics of global economic activity. Furthermore, because all aspects of industrial society use energy, and are themselves constructed using energy, a potentially self-reinforcing feedback exists between energy use and the growth of industrial society.

Our analysis suggests that:

1. By definition, resource distribution networks must fill the space occupied by industrial society. These networks appear to behave near-optimally with respect to minimising energy losses if the space being filled is three-dimensional.

2. Whether optimal or not, the distribution efficiency of the global RADE system declines over time, apparently due to the increasing distribution costs associated with growth-induced network expansion.

3. This declining distribution efficiency appears to be offset by increasing acquisition and end-use efficiencies. This is evidenced by the observed near-constant relative growth rate in energy use that has been maintained at the global scale despite declining distribution efficiencies.

4. The maintenance of growth in energy use at the global scale, specifically at the observed long-term average of $\sim 2.4 \% \mathrm{yr}^{-1}$, may be explained by the minimisation of energy losses over a timescale characteristic of human working lifetimes.

The paper is structured as follows. Section 2 introduces the distribution network theory that underpins the work. This is then used in Sect. 3 to specify and test a predicted scaling relationship between energy flows at the point of acquisition (global primary energy) and those arriving at the points of end-use (global final energy). Section 4 is a discussion on the geometry of the space being filled by the RADE system. Section 5 extends the analysis to consider behaviour at the country scale and how this aggregates to give the observed global-scale behaviour. Section 6 then explores how the observed global trends (at least with respect to primary energy) may extend back to at least 1850 . In so doing we focus on one of the specific mechanisms that appears to mediate the evolution of the RADE system, namely the dematerialisation of resource flows. Section 7 offers a simple model of the full RADE system that accounts for the exponential growth in global energy use observed. This model yields constant relative growth in energy use despite the decreasing returns to scale associated with the expansion of the RADE distribution network(s). Section 8 uses this simple model to attempt to account for the specific observed long-term relative growth rate in global primary energy use of $\sim 2.4 \% \mathrm{yr}^{-1}$. This is done by exploring an optimisation of average personal energy use over specific integration timescales. Finally, Sect. 9 offers some concluding remarks concerning the growth of industrial society in general and some thoughts on further work.

\section{Energy and resource distribution networks}

Resource distribution networks are ubiquitous in nature. Specifically, in biology these networks, such as cardiovascular systems in mammals and vascular systems in higher plants, distribute resources from points of acquisition to the end-use tissues and cells which require these resources to function. Because this form of spatial distribution must itself consume a significant proportion of the acquired energy resources, this has provided strong selective pressure for the evolution of optimal forms of network architecture and operation, with branched directed networks becoming ubiquitous in nature (Savage et al., 2004). Furthermore, biological systems are frequently comprised of complex networks of networks. These networks often co-evolve together as parts of an overall system that both collects and distributes resources, e.g. lungs, blood, lymph and nerves in animals. This means that the networks can be configured both many-toone (i.e. points of acquisition to collection point) and one-tomany (i.e. distribution point to points of end-use) within the same organism. Interestingly, these integrated systems still appear to follow the same theoretical laws, and thus exhibit the same scaling behaviour, as single directed networks (Savage et al., 2004).

We believe it is self-evident that the growth of industrial society has also required the construction of ever-expanding resource distribution networks. These networks include a wide range of infrastructures such as pipes, cables, footpaths, roads, railways, shipping lanes and flight paths. The resources being distributed through these networks are also diverse, including energy, raw materials, manufactured goods, waste, people etc. Here we focus largely on flows of energy. These flows originate from the acquisition of environmentally derived resources which pass through distribution networks to points of end-use. These terminal points of the networks can be thought of as units of energy consumption distributed in the space occupied by industrial society. Taken as a whole, we view this entire process as a RADE system.

RADE networks are optimised by minimising energy distribution losses whilst facilitating resource use (West et al., 1997; Banavar et al., 2010). For energy flows we can define the distribution efficiency of such networks by the ratio of the energy entering the network (primary energy, $x$ ) to that arriving at the points of end-use (final energy, $x^{*}$ ). Networks can be thought of as optimal if, under the constraint of having to satisfy particular end-use demand, the distribution losses, $x-x^{*}$, are minimised for any given $x$ and hence the distribution efficiency can be defined as $x^{*} / x$. Maximisation of this distribution efficiency $\left(x^{*} / x\right)$ can be achieved by both minimising total path lengths and maximising unit path length efficiencies. 
One of the most effective means of minimising path lengths is to optimise the structure of the system by colocating points of end-use at optimal locations within RADE networks. Such behaviour is ubiquitous in industrial society expressed through the process of urbanisation. As for unit path length efficiencies, these can be affected by the method of distribution and the nature of the resource being distributed. Two examples are the increasing use of more fuel efficient vehicles and the liquefaction of natural gas for transportation. It is also important to appreciate that path lengths and their efficiencies are not only determined by infrastructural modes of distribution and the geographies of points of end-use but also by decisions that people make when choosing between the pathways available to them. For example, there may be many routes between two locations, but quicker and less arduous routes are generally preferred.

In summary, our conceptual model of the distribution element of the global RADE system is one of a space-filling network linking points of environmental resource acquisition to points of societal end-use. To explore the possibility that the distribution element of the global RADE system behaves in this way we now investigate the relationship between global primary energy use, $x$, and global final energy use, $x^{*}$. We refrain from looking at the architectures of specific networks because, as stated previously, our analysis is largely dependent on flows of energy at the global scale. As such, we believe it is the emergent behaviour of the network of networks that comprise the global RADE system that is relevant. This suggests that the behaviour of individual network elements must be considered within the context of the other network elements they operate alongside.

\section{Primary and final energy flows at the global scale}

As discussed previously, one definition of an optimal network is where distributional energy losses are minimised. West et al. (1997) employed an optimal model of a fractal space-filling network to demonstrate how distribution networks in nature can give rise to observed scaling patterns. Banavaar et al. (2010) showed that these patterns were not restricted to fractal networks. Although not articulated in these papers, both of these analyses allude to a theoretical upper limit of the distribution efficiency $x^{*} / x$ for any given space being occupied by a distribution network. If $L$ is the linear size of the network then the size of the space being filled by the network is given by $L^{D}$ where $D$ is the dimension of the space being filled by the network. Independent of the specific modelling assumptions considered by either West or Banavaar, to be consistent with their modelling results, optimal network efficiency has to scale with network size according to $x^{*} / x \propto L^{-D /(D+1)}$. This even holds as $L$ tends to zero because $D$ must also tend to zero in the limit, so the efficiency of the network has a theoretical unity upper limit even as $L \rightarrow 0$.
The scaling relationship between $x^{*} / x$ and $L$ suggests that the relationship between the energy arriving at the points of final use, $x^{*}$, and the primary energy flow entering the network, $x$, should scale as $x^{*} \propto x^{D /(D+1)}$. This is the same scaling relationship proposed by Dalgaard and Strulik (2011), building on Banavar et al. (2010), when attempting to account for the energy distribution losses in the US electricity grid. The reason for sub-unity scaling between $x^{*}$ and $x$ is simply because as the size of the system increases so does its average path length between points of acquisition and end-use, $L$. This increase in path length causes the distribution efficiency, $x^{*} / x$, to fall. However, rather than the efficiency falling in proportion to increases in network size, $L^{D}$, it falls in proportion to $L^{D /(D+1)}$, i.e. at a rate slower than one would predict from geometric considerations alone. This is because of the optimisation of the distribution links within the RADE network as discussed earlier.

We define global primary energy use, $x$, as the annual energy flow from nature to society in the form of wood, coal, oil, gas, nuclear, renewables and food. Primary energy is generally treated as the combustible energy equivalent of these sources. This does introduce some complexity when handling non-combustible sources (e.g. wood used for construction), but given that these are such a small fraction of the total this is not believed to significantly affect the quality of the aggregate global primary energy data (Macknick, 2009). Total food use was estimated by assuming global per capita consumption of $3 \times 10^{9} \mathrm{~J} \mathrm{yr}^{-1}$ (United Nations, 2002), although presently this represents less than $1 \%$ of the total.

We define $x^{*}$ (final energy use) as the energy available to industrial society once distribution losses have been accounted for. The International Energy Agency (IEA) provides estimates of energy lost through its acquisition, processing and delivery to end users. However, these data do not account for energy losses associated with either the acquisition of non-energy resources and agriculture or the transport of all mass through industrial society. In an effort to account for these losses to obtain $x^{*}$ we have subtracted the IEA estimates of energy used in quarrying, mining, agriculture, forestry and, in particular, transport, from the IEA final energy consumption data.

Considering transport as a distributional loss raises an important conceptual issue. Currently approximately $50 \%$ of transport energy use is associated with passenger movements. Traditionally these are seen as end-use energy services enjoyed by people. However, here we treat them as necessary distributional losses required to get energy consumers to spatial nodes where they can contribute to the continued growth of the RADE system. In other words, we view the flow of people like the flow of any other mass in the RADE system. Hence we view nodes of final consumption as static locations where final energy is consumed, albeit with human agency applied to the purpose of consumption. Importantly, this means that nodes are best viewed as more than just passive recipients of resource. Instead final consumption nodes 
must in turn facilitate the further acquisition of resources through extending the interface between industrial society and the environment (Garrett, 2011, 2012). Taking this approach essentially means that all components of the RADE system that are mobile are distributional and all static components are either acquiring resources or consuming resources for end-use. Although this framing may be contrary to more traditional views of humans as "energy consumers" we believe it is at least internally consistent with our view of the RADE system and the role of humans in it.

It should be noted that our estimates of $x^{*}$ do not adequately account for the distribution energy losses occurring between the point of sale and the point of end-use of energy (e.g. in the case of electricity, losses occurring between the meter and the plug). However, we assume these to be relatively small relative to all upstream losses associated with acquiring and distributing all resources. The small underestimate of distributional loss implied by our estimate of final energy using the IEA data should be partially offset by the fact that our revision of the IEA final energy use will also include some non-distributional energy uses (e.g. end-use energy in agriculture) due to the way the IEA data are compiled.

We define the space associated with unit final energy consumption (referred to as a "control volume" by Dalgaard and Strulik, 2011) as being that where the consumption of useful energy in that space is significantly greater than the transfer of useful energy from that space to other regions of the network. These spaces are complex entities and not easy to identify, because in a global mean sense they are comprised of broad portfolios of energy uses. That said, examples of end-use processes might include reading this article on a computer, cooking, constructing or demolishing a residential building etc.

As for energy losses due to energy transformations that occur between primary and final energy, these are far more significant. One way of reconciling these transformations within the current framework is that they are deployed to reduce mass flows in critical parts of the system (e.g. by generating electricity from coal). Here the substantial energy losses incurred by these transformations are presumably offset by the downstream savings they facilitate (in this example, by reducing the amount of coal distributed to individual households). This point will be explored in greater detail in Sect. 4 .

Figure 1a shows the relationship between $x$ and $x^{*}$ for the available IEA data (IEA, 2012). We find that $x^{*} \propto x^{c}$ $(c=0.75 \pm 0.02)^{1}$, i.e. the scaling exponent $c$ is statistically indistinguishable from three quarters. For reference, using the IEA definition of final energy gives $c=0.84 \pm 0.01$ with

\footnotetext{
${ }^{1}$ Scaling exponents have been estimated using ordinary least squares of the linear model $\ln \left(x^{*}\right)=\theta_{1} \ln (x)+\theta_{2}$. Parameter uncertainties are reported at $95 \%$ confidence. $1 \sigma$ uncertainties in the data were assumed to be $5 \%$ (Macknick, 2009). All results were also cross-checked using nonlinear least squares of the untransformed data.
}

practically all of the difference between these two estimates attributable to the inclusion of transport in our specification of final energy. Figure $1 \mathrm{~b}$ shows the equivalent relationship between the distribution efficiency, $x^{*} / x$, and primary energy, $x$. It confirms that, as predicted, the overall efficiency of the network has progressively fallen over time as $x$ has increased and is now below $50 \%$, i.e. more than half of all primary energy is now used simply to move all the materials and resources required by industrial society (e.g. environmentally derived materials, mobile system infrastructure and people) to final nodes of end-use.

\section{What space does society inhabit?}

The fact that we observe scaling between $x^{*}$ and $x$ that is statistically indistinguishable from three quarters suggests $D=3$ in the framework set out above. Although the relative dimensions are far from equal, it is self-evident that the networks moving mass through global industrial society occupy a three-dimensional space. However, since the horizontal dimensions of this space are approximately 3 orders of magnitude greater than the vertical dimension (delineated by, for example, the distance between the deepest mines and the height at which aircraft fly), it is appropriate to ask whether this space is more appropriately approximated by a twodimensional surface rather than a three-dimensional volume. This question cannot be answered conclusively here but we offer the following lines of evidence to suggest that $D=3$ does indeed provide a plausible description of the space filled by the global RADE system.

Firstly, the effect of gravity obviously imposes disproportionately higher distribution costs on movement in the vertical dimension than in the horizontal. We conjecture that these differences in cost are between 1 and 2 orders of magnitude. This could rise significantly above 3 orders of magnitude when the engineering difficulties of exploring the vertical dimension below ground are considered. Whether this is sufficient to result in $D=3$ in the global RADE networks is unclear although we note that we invariably treat the atmosphere as a three-dimensional object even though it too has a severely diminished vertical dimension. Secondly, the scaling behaviour of urban centres suggests that people occupy a three-dimensional space at the city scale, despite the fact that the vertical dimension is again very much attenuated (Nordbeck, 1971). Even silicon chips, which have a trivial vertical dimension, exhibit scaling of the order of $D=2.5$ (Deng and Maly, 2004) suggesting that even a highly attenuated vertical dimension with no disproportional losses can result in nontrivial scaling effects. Finally, although the Earth's surface can, by definition, be considered a two-dimensional object, the curvature of this surface at the global scale may be sufficient to introduce three-dimensional effects in the links between network nodes. 


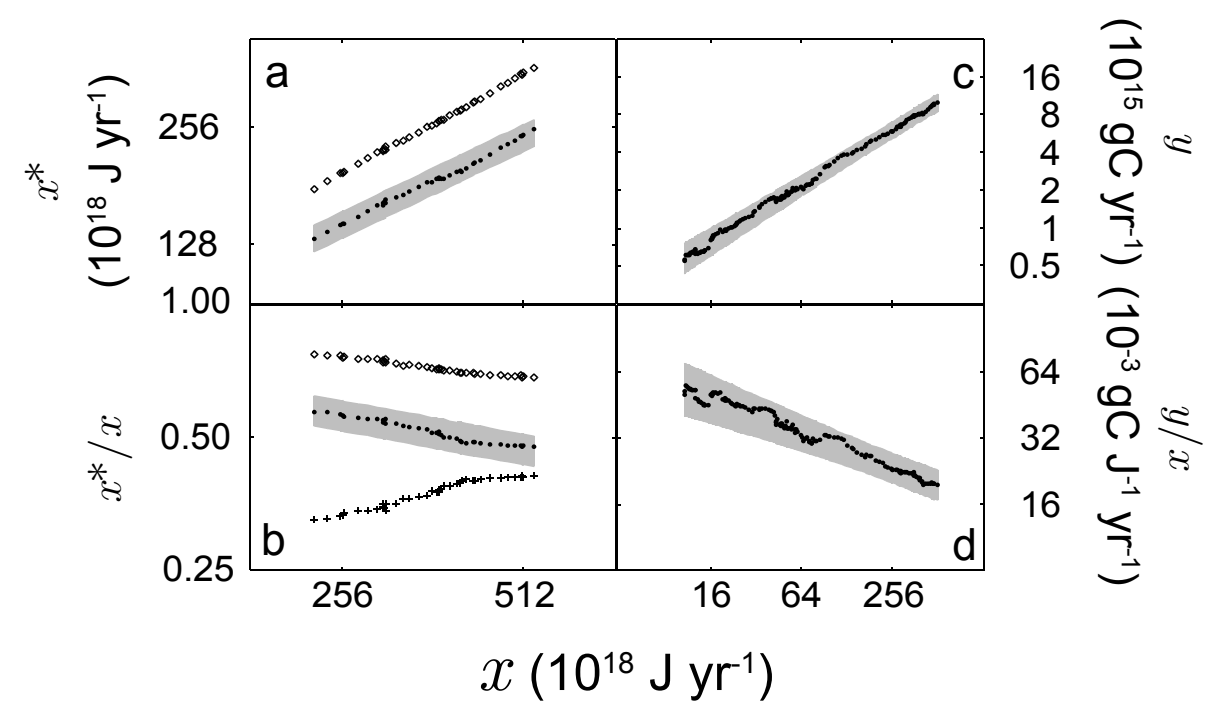

Figure 1. (a) The relationship between global primary energy use, $x$, and global final energy, $x^{*}$. Two definitions of final energy are shown; (o) are the IEA estimates, $(\bullet)$ are the IEA estimates adjusted for energy used for transport, agriculture, forestry, mining and quarrying. (b) The relationship between global primary energy use, $x$, and primary to final network efficiency, defined as the ratio $x^{*} / x(\bullet)$. Also shown are the estimated variations in end-use efficiency assuming a total system efficiency $10 \%(+)$. The IEA definition of primary to final energy efficiencies (o) are also shown for reference. (c) The relationship between global primary energy use, $x$, and global anthropogenic $\mathrm{CO}_{2}$ emissions, $y$, for the data shown in Fig. 3. (d) The relationship between global primary energy use, $x$, and the carbon intensity of global primary energy, $x / y$ again for the data shown in Fig. 3. The bands for all plots represent 5 th to 95 th uncertainty ranges from the linear regressions. See text for all data sources and compilation.

An alternative explanation to our observed scaling behaviour of the global energy system is that $D<3$ and that the system operates supra-optimally, which appears infeasible. Equally, the observed exponent of three quarters may have arisen by chance and the systemic explanation explored here is incorrect. This proposition cannot be rejected, but then neither can the proposition that $D=3$. It also seems somewhat anomalous that we would observe a scaling exponent that is indistinguishable from three quarters if the system was twodimensional.

If the global RADE network has the dimensions of $D=3$, then the scaling observed between $x$ and $x^{*}$ suggests that, at the global scale, the distribution networks that underpin the RADE system are, in aggregate, optimised with respect to energy losses, despite filling a highly irregular threedimensional space. That the RADE networks created within industrial society should be near-optimal does not seem unreasonable given the pressures to seek out performance improvements in a competitive global market system.

As a result of the framework set out above we identify three related mechanisms through which distribution efficiency gains, and hence the optimisation of this element of the global RADE system, could be realised.

1. The efficiency of network infrastructure is progressively improved over time (e.g. by the use of more aerodynamic vehicles, more efficient combustion processes).
2. The flows are themselves persistently dematerialised over time (e.g. by introduction of lighter vehicles, shifting the primary fuel mix from wood to coal to oil to gas or turning coal into electricity - see later).

3. The structure of and practices on the network are modified over time to reduce average path lengths, $L$ (e.g. by building a new road, introducing car navigation systems, by the reorganisation of the points of acquisition and end-use during urbanisation).

The first two of these are primarily concerned with maximising unit path length efficiencies, whilst the third is primarily concerned with minimising total path lengths. It may also be that processes like urbanisation offer additional benefits in that the increased social interactions that result from the clustering of people stimulate the innovations required to discover and realise the three efficiency mechanisms mentioned above (Bettencourt, 2013). These innovations have to be continuously discovered, developed and implemented in order to accommodate the growth of the RADE system. We shall return to the subject of resource flow dematerialisation in more detail in Sect. 6.

\section{What happens at the regional scale?}

Thus far our analysis has been focused at the global scale, yet this global behaviour must emerge from regional-scale dynamics. Each region, $i$, uses primary energy, $x_{i}$, and final 
energy, $x_{i}^{*}$, where $\sum x_{i}=x$ and $\sum x_{i}^{*}=x^{*}$. As we have already discussed, networks tend to become less efficient as they expand due to the size-related penalties of growth. It appears that this behaviour is observed at the global scale with $x^{*} / x$ decreasing as $x$ increases (Fig. 1b). In the absence of further innovation and all else remaining equal, we would anticipate the same behaviour at the regional scale. This means that in portions of the system with higher energy use densities (i.e. higher energy use per unit space) we would expect lower regional distribution network efficiencies, $x_{i}^{*} / x_{i}$. Conversely, in portions of the system with lower energy use densities (i.e. lower energy use per unit space) we would anticipate higher regional distribution network efficiencies. However, if this divergence in distribution efficiencies between regions, due to differing energy use densities, actually arose at any given point in time it would presumably cause the global system to be sub-optimal because global final energy use could be increased for the same global primary energy use simply by shifting resource distribution from the less efficient to the more efficient portions of the system.

This sub-optimality is not what we observe at the global scale. Instead, as discussed above, the observed approximate three quarter scaling between $x$ and $x^{*}$ indicates that the global RADE system is operating near-optimally with respect to distribution if $D=3$. Because it appears that the system could be near-optimal at the global scale, we would expect distribution efficiency gains to be persistently sought out. In other words, if optimal, the RADE system would evolve such that it seeks to exhaust all potential improvements with respect to energy use. As a result, we hypothesise that, at any particular point in time, all countries of the world should have similar network efficiencies and these should be independent of their energy use densities (i.e. their $x_{i}$ per unit space). In order to achieve this, countries located in more energy-dense (i.e. more developed) portions of the system presumably innovate more aggressively on distribution efficiency to overcome the size-related penalties of growth than do those in less energy-dense (i.e. less developed) portions of the system. Once again, examples of these innovations might be the enhanced efficiency of mass transport, enhanced urbanisation and the enhanced use of gas or electricity.

We test this hypothesis using IEA data for 140 countries for the period 1971-2010. Figure 2 shows the relationship between primary and final energy use $\left(x_{i}\right.$ and $\left.x_{i}^{*}\right)$ for these data. In the absence of a measure of the effective volume being filled by society, we have normalised energy use by country land area in order to attempt to reflect the space-filling aspect of the system. Because this assumes uniform average vertical dimensions between countries and is applied to both $x_{i}$ and $x_{i}^{*}$ this only changes the relative positions of countries, not their individual efficiencies.

As predicted, Fig. 2 shows that at any given point in time $x_{i}^{*} / x_{i}$ is largely independent of $x_{i}\left(x_{i}^{*} \propto x_{i}^{c} ; c=0.97 \pm 0.03\right.$ for all 40 years). This appears to hold across all 140 countries sampled, which have a range of $10^{5}$ in energy use per

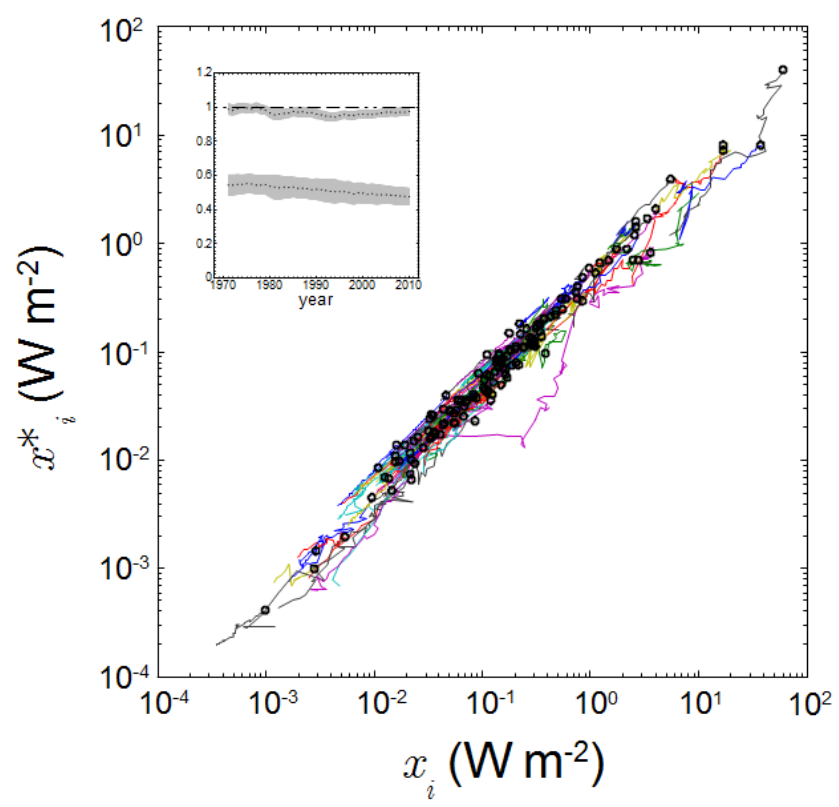

Figure 2. The relationship between country-specific primary energy use, $x_{i}$, and final energy use, $x_{i}^{*}$ for the period 1971-2010. Individual countries are marked with different colours, $N=140$. The data for all countries for 2010 are marked separately (o). All country-specific energy data are normalised using the surface area of the country. The surface area is an imperfect proxy for the space occupied by each country if the global system is filling a threedimensional volume. In the absence of data, we assume that the magnitude of the vertical dimension is constant across all 140 countries. Note that the higher per unit area energy consumers have per unit area energy flows that are a significant proportion of the solar constant. The inset figure shows both the exponential scaling coefficient estimated from the annual relationship between $x_{i}$ and $x_{i}^{*}$ (values near 1 ) along with the primary-to-final energy efficiency $x_{i}^{*} / x_{i}$ plotted for each year 1970 to 2010 . The bands represent 5 th to 95th uncertainty range for the estimates. See text for data sources and compilation.

unit area. For example, currently the UK has a similar distribution efficiency, $x^{*} / x$, to that of Bolivia (0.473 vs. 0.466), despite having $>10^{2}$ greater energy use density. A significant contributor to the variation in $x_{i}^{*}$ and $x_{i}$ is probably the less reliable IEA energy data for less-developed countries. We note that the variation created by these uncertainties is not systematically above or below the central trend. Moreover, we would expect the relationship to be even clearer if we were able to normalise the data by the appropriate volume, rather than area, occupied by society in each country.

Because of the apparent invariance of distribution network efficiency with energy use density it would appear that regional networks are not scaled versions of the global system, i.e. the global RADE network appears to be scale dependent rather than scale free. This implies that you cannot simply look at isolated sub-components of the global RADE 
network (e.g. individual countries) in order to infer the behaviour of the global system.

\section{Long-run growth and decarbonisation of global energy use}

Thus far we have focused on data on primary and final energy use covering the last 40 years. However, there are data on primary energy use going back much further than this. As mentioned earlier, global primary energy use, $x$, is taken here to be the annual energy flow from the environment to society in the form of wood, coal, oil, gas, nuclear, renewables and food. In order to construct a consistent time series for $x$ since 1850, following Jarvis et al. (2012), the global primary energy use data for the period 1850 to 1964 are taken from Grübler (2003) and for the period from 1965 to 2010 from BP (2011). We note that compiling long-term historic series for virtually any relevant measure of economic activity is challenging due to the paucity of available data and increasing uncertainties the further back one goes. Data on energy use are not exempt from these limitations. For example, the Grübler data we use do not appear to capture the full portfolio of renewables in use in the 1800 s (e.g. wind and water power). However, we also note that the energy data used here still represents one of the best observed metrics of global economic activity. Also on the specific issue of renewables post-1850, evidence suggests that they constituted a negligible part of the global energy portfolio during this period (O'Connor and Cleveland, 2014; Fouquet, 2014).

We opt to use the BP data in order to attempt to have some limited independence from the IEA data used to explore the relationship between $x$ and $x^{*}$. To produce a homogeneous record for 1850 to 2010 the mean difference between the two series for the period 1965 to 1995 (which is largely due to lack of wood fuel use in the BP data set) was added to the BP data. The data were converted from tonnes of oil equivalent (toe) to Joules, assuming $10^{18} \mathrm{~J}=2.38 \times 10^{7}$ toe (Sims et al., 2007).

Figure 3 shows the primary energy use data, $x$, for the period 1850-2010. These suggest that, in the long term, $x$ has grown near exponentially since at least 1850 , with a longterm relative growth rate of $2.4( \pm 0.08) \mathrm{yr}^{-1}$ (Jarvis et al., 2012). ${ }^{2}$ Using global Gross Domestic Product (GDP) data as a proxy for global energy use, Garrett (2014) suggests that the relative growth rate of global primary energy has

\footnotetext{
${ }^{2}$ Relative growth rates have been estimated using ordinary least squares of the general linear model $\ln (x)=\theta\left(t-t_{1}\right)$. Parameter uncertainties are reported at $95 \%$ confidence. The model residuals, which were significantly autocorrelated, have been de-correlated assuming a first-order autoregressive noise model to minimise any bias in the estimates of $\theta .1 \sigma$ uncertainties in the data were assumed to be $5 \%$ in energy use and fossils fuel emissions (Macknick, 2009); and $20 \%$ in land-based emissions (C. Le Quéré, personal communication, 2013).
}

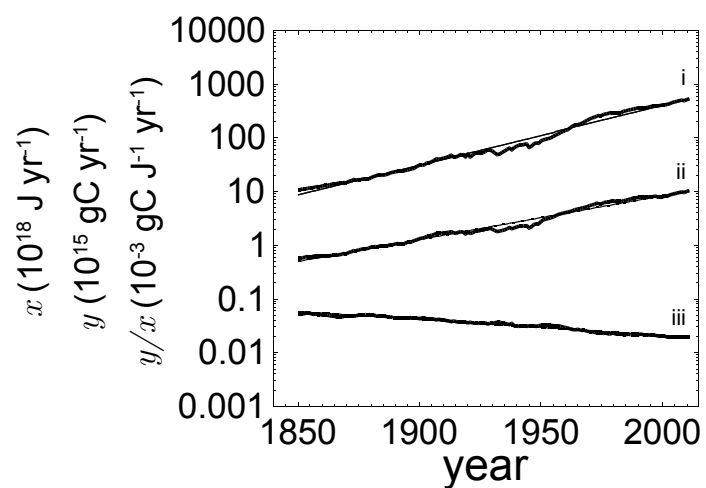

Figure 3. (i) Annual global primary energy use [11, 12, 13] with regression line given by $\ln x=a\left(t-t_{1}\right) ; a=0.0238 \pm 0.0008 \mathrm{yr}^{-1}$; $t_{1}=1775 \pm 3.5 \mathrm{CE}$. (ii) Annual global anthropogenic $\mathrm{CO}_{2}$ emissions $[15,16,17]$ with regression line given by $\ln y=b\left(t-t_{1}\right)$; $b=0.0179 \pm 0.0006 \mathrm{yr}^{-1} ; t_{1}=\mathrm{AD} 1883 \pm 1.7$. (iii) Carbon intensity of global primary energy determined by the ratio $y / x$. See text for data sources and compilation.

increased significantly over this period. The data and analysis in Fig. 3 would indicate otherwise, although clearly there are significant uncertainties over actual global primary energy measures both now and more significantly pre-1900. For example it is unclear what contribution wind makes through shipping over this period. That said, that the long-run growth in primary energy use observed over the last 40 years actually appears to extend back at least 160 years suggests that the processes and trends that have underpinned the development of the global RADE system may have actually been operating for considerably longer than the IEA data provide evidence for. If this is the case we would predict that the optimisation mechanisms identified earlier would also have been at work over the same period. In particular, we would expect that these optimisation mechanisms would be sought out and implemented at a rate that matches the growth-induced declines in distribution efficiency experienced by the global RADE system revealed in Fig. $1 b$.

To explore this proposition we focus on the dematerialisation of resource flows. The primary energy carrier for industrial society is carbon, and in fact some estimates suggest that carbon currently accounts for as much as $50 \%$ of the total amount of materials moved by industrial society through its RADE networks (Dittrich and Bringezu, 2010). This material flow ultimately leads to the emissions of carbon dioxide as carbon-based energy carriers are consumed. Hence the emission rates of carbon dioxide can be seen as giving a measure of the flow of carbon-based energy carriers through the RADE system. In the context of the distribution costs of resources, decarbonisation can therefore be viewed as merely one, albeit important, component of a general systemic dematerialisation of resource flows (Ausubel, 1989) through the RADE system. Here dematerialisation is taken as the removal of "unnecessary" mass from resource 
flows through innovation. This systemic dematerialisation is almost certainly not unique to carbon and may indeed be a necessary response to the increasing distribution costs inherent in any expanding network.

To estimate the amount of carbon flowing through the RADE system we use global carbon emissions data from Houghten (2010), Boden et al. (2010) and Peters et al. (2012). ${ }^{3}$ Figure 3 shows that global carbon emissions, $y$, have also grown near-exponentially since at least 1850 at the long-term rate of $1.8( \pm 0.06) \% \mathrm{yr}^{-1}$ (Jarvis et al., 2012). The difference between this growth rate and the growth rate of primary energy indicates that the global primary energy portfolio has been systematically decarbonised at a rate of $\sim 0.6 \% \mathrm{yr}^{-1}$ since at least 1850 (Jarvis et al., 2012). This decarbonisation is normally viewed as being the result of societal preferences for cleaner, more convenient, energy carriers (Grübler and Nakienovic, 1996). It has also been partially attributed to improvements in the efficiency of converting solid, liquid and gaseous fuels to electricity (Nakienovic, 1993). Both these explanations seem unsatisfactory given the constant long-run nature of the decline in carbon intensity. Furthermore, conversion efficiency affects the distribution efficiency, $x^{*} / x$, and hence $x^{*}$. It does not directly affect the primary portfolio comprising $x$. Instead, it is more appropriate to consider innovations on energy transformations as co-evolving with the portfolio of global primary energy. More specifically, it appears to us that the pattern of decarbonisation of the global energy portfolio is in line with, and a necessary response to, the declining distribution efficiency of the global RADE network, $x^{*} / x$.

The long-term exponential growth in both $x$ and $y$ set out above suggests that global primary energy use and carbon flows share a common exponential scaling relationship, $y \propto x^{b / a}$, where $a$ and $b$ are the relative growth rates of $x$ and $y$, respectively. Figure 1c shows the scaling relationship between $x$ and $y$ since 1850. From these data we see that the exponential scaling between $x$ and $y$ is not only a property of the 160 year average behaviour, but also holds remarkably well on intervening timescales. This relationship has a scaling exponent of $b / a=0.76( \pm 0.05)$. Calculating this exponent using the long-term (160 year) exponents for $x$ and $y$ gives $b / a=0.75( \pm 0.06)$. As with the primary-to-final scal-

\footnotetext{
${ }^{3}$ As in Jarvis et al. (2012), we have included land use change in the measurement of carbon emissions because our definition of $x$ necessarily includes wood use. However, although deforestation dominates the land use change emissions estimates, not all deforestation emissions are associated directly with the production and distribution of wood as a fuel, as they include significant contributions from slash-and-burn land clearance activities for food production. Furthermore, carbon-neutral biomass production is not accommodated by net anthropogenic $\mathrm{CO}_{2}$ emissions inventories. Between 1850 and 1900 wood fuel use constituted a significant proportion of global primary energy use (Grubler, 2003) but beyond 1900 their contribution to global carbon use quickly become dominated by fossil fuels.
}

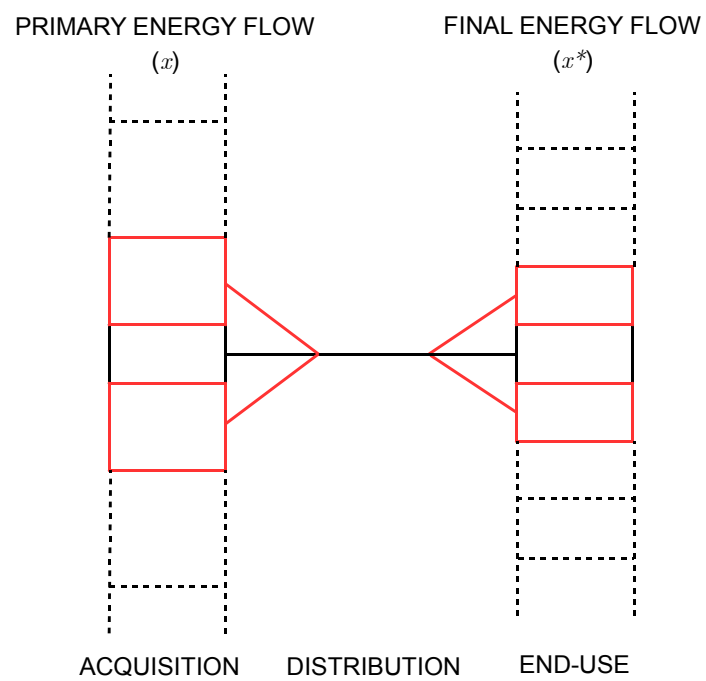

Figure 4. A schematic 1-D representation of the global RADE system. Here units of primary energy, $x$, are linked to those of final energy, $x^{*}$, via a distribution network. The black outlined system represents the initial stage of the systems evolution. The red outlined system represents the subsequent addition of units of final energy use and hence primary energy use and hence the expansion of the network linking the two.

ing identified earlier, this too is statistically indistinguishable from three quarters.

The scaling observed between $x^{*}$ and $x$ and between $y$ and $x$ therefore leads to direct proportionality between carbon intensity and network distribution efficiency $\left(y / x \propto x^{c} x^{*} / x\right.$; $c=-0.006 \pm 0.043$, hence $x^{c} \approx 1$; see Fig. $2 \mathrm{c}$ and d). As predicted then, the implementation of dematerialisation appears to occur at a rate that is proportional to the growth-induced declines in distribution efficiency experienced by the global RADE system. This would appear to further corroborate our view of the role of the distribution networks that make up the global RADE system. Interestingly, the result of the scaling between $x, x^{*}$ and $y$ also indicates that total global anthropogenic $\mathrm{CO}_{2}$ emissions grow in proportion to the consumption of final energy, $x^{*}$, not primary energy, $x$.

To place our interpretation of the role of decarbonisation of the primary fuel mix in context, the historic trend in primary energy use from wood to coal to oil to gas and renewables has occurred because it has allowed less mass to be transported through the RADE network per unit of energy used (Ausubel, 1989). Fundamentally this represents an innovation on the distribution efficiency, $x^{*} / x$.

The recent shift towards the use of gas globally (ExxonMobil, 2013) represents a particularly interesting continuation of this trend. Gas has a lower unit volume energy density than other fossil fuel sources (i.e. coal or oil). Lower energy density carriers like gas suffer from higher longdistance transportation costs, which is presumably why a smaller proportion of gas is traded internationally than oil 
or coal (ExxonMobil, 2013). However, gas also incurs lower energetic costs when being distributed though the more tortuous finer terminal parts of the distribution network (Banavar et al., 2010).

To illustrate this point it is useful to consider the paths that make up the global distribution network as passing through three stages: the gathering together of resources from their extraction points in the environment; the intermediate transportation of resources from regions of extraction to regions of end-use; and lastly the distribution of resources to the nodes of final end-use (see Fig. 4). As the global distribution network develops, the relative importance of these three network elements in controlling overall distribution costs should change. This is because, although the long-distance intermediate costs increase as the network expands, the final distribution costs increase faster (Banavar et al., 2010). This concept is already well established in transportation and telecommunications networks as "the last mile problem". So as the RADE system as a whole grows, low carbon energy carriers such as gas are increasingly preferred, and this preference is most keenly felt in the final distribution elements of the RADE system. This seems intuitive when one imagines the vastly increased distributional costs that an advanced (i.e. energy dense) country like Germany would incur if it tried to meet its energy demands for heating and cooking solely through distributing coal to individual end users, instead of by the increasing use of gas.

This demand for low-carbon energy carriers in the terminal parts of the RADE system may also stimulate innovations such as the liquefaction of natural gas (LNG) because LNG reduces the costs of moving gas long distances during intermediate transportation. Similarly, innovations in hydraulic fracturing can allow the exploitation of gas resources near to the final point of use, removing some of the need for longdistance transport. Lastly, electrification is currently the primary means of dematerialising energy flows through transformation and, just as with gas, the lower energetic costs of transmitting electricity are most effectively deployed in the final distribution parts of the network, e.g. in developed, urbanised areas. This would explain why decarbonisation is sometimes associated with energy transformation efficiencies given that both would co-evolve as distribution networks expand. However, we would argue that it is misleading to implicate conversion efficiency as a driver for the decarbonisation of energy portfolios. It is interesting to note from Fig. 3 that the recent increase in global coal use, which tends to counter the long-term trend of decarbonisation, has been largely offset at the global scale by the increased use of gas, renewables and decreases in land-based emissions. Furthermore, the vast majority of this coal is not distributed to final points of end-use as it was a century ago. Instead it is used to generate electricity which is then distributed to end users, which is consistent with the process of dematerialisation discussed above.

\section{Total energy efficiency and growth - a model}

If industrial society does indeed experience declining network distribution efficiency, as indicated by Fig. 2b, then, all else remaining equal, global industrial society should experience size-related limits to growth in $x$, just as growth is self-limiting in most biological systems (West et al., 2001). It is possible that the observed long-term exponential growth in $x$ could reflect the early stages of what is otherwise logistic size-restricted growth. If this is the case then ultimately the growth of the global RADE system would be self-limiting, even though primary energy use has risen exponentially and by $\sim 50$-fold since 1850 . This in and of itself is a fascinating prospect.

However, we argue that global industrial society is continually innovating to overcome the increasing size-related penalties associated with growth. This seems consistent with the apparent growth imperative of industrial society and the fact that the observed declines in distribution efficiency shown in Fig. 1b have been countered in order to maintain the near-constant relative growth rate of $\sim 2.4 \% \mathrm{yr}^{-1}$ shown in Fig. 3. We illustrate this point with the following simple endogenous growth model in which we treat global industrial society as a homogeneous unit.

As global society grows, it acquires additional primary energy flows to support additional end uses, the two being linked by extensions to existing networks. Therefore, we can conceptualise the growth of industrial society both as its expansion into new environmental resources, and hence space, and the establishment of new points of end-use. Although the space occupied by industrial society is complex, if $D=3$, then it is appropriate to consider society as occupying an (irregular) volume, $V$. If the end-use control volumes are considered as being within $V$ then, from a network perspective, it is also reasonable to assume the in-use environmental resources are also within $V$, i.e. industrial society grows into its resources (Garrett, 2011). If so, then we assume in the simplest case that the flow of resource into industrial society is proportional to the volume of resources subsumed and hence $V .{ }^{4}$ Therefore, in the absence of any storage, the supply and consumption of primary energy resources might simply be described by

$x=k_{\mathrm{A}} V$,

where the proportionality $k_{\mathrm{A}}$ is the resource acquisition efficiency and is the product of the energy potential between the environmentally derived energy resources and society and the efficiency with which these resources can be assimilated into the RADE system and hence into industrial society.

\footnotetext{
${ }^{4}$ We note that Garrett (2014) assumes environmental resources flow to industrial society across an environment-society interface (surface) and hence speculates that this flow is proportional to $V^{1 / 3}$ on theoretical grounds.
} 
Assuming networks distribute captured resources optimally within the volume, $V$, then the final energy flow arriving at points of end-use, $x^{*}$, is given by

$x^{*}=g x^{D /(D+1)}$,

where $g$ is a scaling constant (Dalgaard and Strulik, 2011). Once at the points of end-use, and after subtracting the enduse inefficiencies (i.e. the costs of transforming final energy into useful work), the remaining portion of $x^{*}$ provides work which is used to increase the size of industrial society (Garrett, 2011, 2012). This in turn expands $V$ and allows the cooption of further resources. Because it requires work to expand $V$, the size of industrial society can also be viewed as the accumulation of this work, $X$, occupying the space, $V$. The balance of this accumulated work can be seen as the difference between work done and the decay of the stock of accumulated work,

$\frac{\mathrm{d} X}{\mathrm{~d} t}=k_{\mathrm{E}} x^{*}-k_{\mathrm{D}} X$,

where $k_{\mathrm{E}}$ is the end-use efficiency of final energy conversion to useful work and $k_{\mathrm{D}}$ is the aggregate decay rate of $X$.

Equations (1)-(3) are exponential in $x$, in line with the observations in Fig. 3, if $X \propto V$, i.e. work operates uniformly in space. Because the mean energy density of industrial society is unknown we assume $X=V$ for simplicity given this has no bearing on our analysis. Equations (1)-(3) now give

$\frac{\mathrm{d} x}{\mathrm{~d} t}=\left(k_{\mathrm{A}} k_{\mathrm{E}} g x^{-1 / 4}-k_{\mathrm{D}}\right) x=a x$,

where $a$ is the relative growth rate of global primary energy, or $\sim 2.4 \% \mathrm{yr}^{-1}$. From Eq. (4) we see that $a \propto x^{-1 / 4}$ (West et al., 2001), i.e. as the system grows the relative growth rate should fall. Therefore, in order to maintain exponential growth in $x$, the acquisition efficiency, $k_{\mathrm{A}}$, and/or the end-use efficiency, $k_{\mathrm{E}}$, must be increased and/or the decay rate, $k_{\mathrm{D}}$, must be decreased to compensate for the declining capacity of primary energy to support growth.

We assume that both $k_{\mathrm{A}}$ and $k_{\mathrm{E}}$ are dynamically adjusted by society in order to maintain growth, whilst $k_{\mathrm{D}}$ remains fixed. The assumption of a fixed decay rate is supported by the observation that the mean lifetime of technologies (Grübler et al., 1999), including large energy projects (Davis et al., 2010) has remained fairly constant at $\sim 40$ years, or $\left(\sim 2.4 \% \mathrm{yr}^{-1}\right)^{-1}$, i.e. technologies decay at the same rate as the relative growth of industrial society $\left(k_{\mathrm{D}}=a\right)$. One way of understanding such a link is that physical capital is turned over at about the same rate as the system evolves, thereby allowing the appropriate rate of adoption of the innovations required to preserve growth at the rate $a$.

In the absence of any change in the acquisition and end-use efficiencies, $a \propto x^{-1 / 4}$. Therefore for $a$ to remain constant requires
$k_{\mathrm{A}} k_{\mathrm{E}}=h x^{1 / 4}$,

where again $h$ is a scaling constant. This now gives exponential growth in $x$ as

$\frac{\mathrm{d} x}{\mathrm{~d} t}=\left(h g-k_{\mathrm{D}}\right) x=a x$

and $h g=2 a$ if $k_{\mathrm{D}}=a$ as discussed above. Within this framework, if $k_{\mathrm{D}}=a$, the energy that is available to grow $X$ and hence $V, x_{\mathrm{G}}$, is given by

$x_{\mathrm{G}}=k_{\mathrm{A}}^{-1} h g x=\varepsilon x$,

where $\varepsilon$ is the overall primary to end-use energy efficiency of the RADE system (see also Garrett, 2011). The observed near-constancy of the long-term relative growth rate in global primary energy use strongly suggests that $\varepsilon$ has remained more or less constant over at least the last 160 years. Using IEA data, Nakicenovic et al. (1996) have estimated $\varepsilon$ to be $\sim 30 \%$, although this figure is highly uncertain because their analysis could not accurately account for the end-use efficiency of final energy in productive work. Ayres (1989) attempted a similar analysis for the US attempting to account for so-called useful work (or exergy) effects and derived an estimate of $2.5 \%$ for $\varepsilon$. In addition to the declining network distribution efficiency $x^{*} / x$, Fig. $1 \mathrm{~b}$ also shows an illustration of the simultaneous increases in end-use efficiencies, $k_{\mathrm{E}}$, required to keep $\varepsilon$ at a hypothetical value of $10 \%$, assuming $k_{\mathrm{A}}$ is constant. ${ }^{5}$ Figure 5 shows the model described above in block diagram form.

\section{Growth optimisation and working lifetimes}

Thus far we have sought to illustrate how the growth of industrial society, as determined by its energy use, could be controlled by the optimisation of the RADE network. In part this optimisation is facilitated by reducing material flows including decarbonisation of the primary energy portfolio. We have also attempted to show that, despite this optimisation, RADE network efficiency necessarily falls. We have therefore set out how an observed near-constant relative growth rate is maintained through continuous but measured implementation of innovations on both energy acquisition and enduse efficiencies. An important question that remains is, if growth is desirable, why does industrial society only compensate for falling distribution network efficiency, and not overcompensate to allow super-exponential growth? Or, put another way, why is constant relative growth good? This cannot be due to the lack of innovative capacity because there appears to be a surplus of this available to enhance acquisition

\footnotetext{
${ }^{5}$ Here $10 \%$ is simply taken as an illustrative value for $\varepsilon$ given its true value remains highly uncertain. This only affects the level of the relationship between $x_{\mathrm{G}} / x^{*}$ and $x$, not its scaling. Having assumed this value we can also specify a fixed value for $k_{\mathrm{A}}$ from Eq. (7) of $2 a / 0.1=0.5 \mathrm{yr}^{-1}$ for the case of $X=V$.
} 


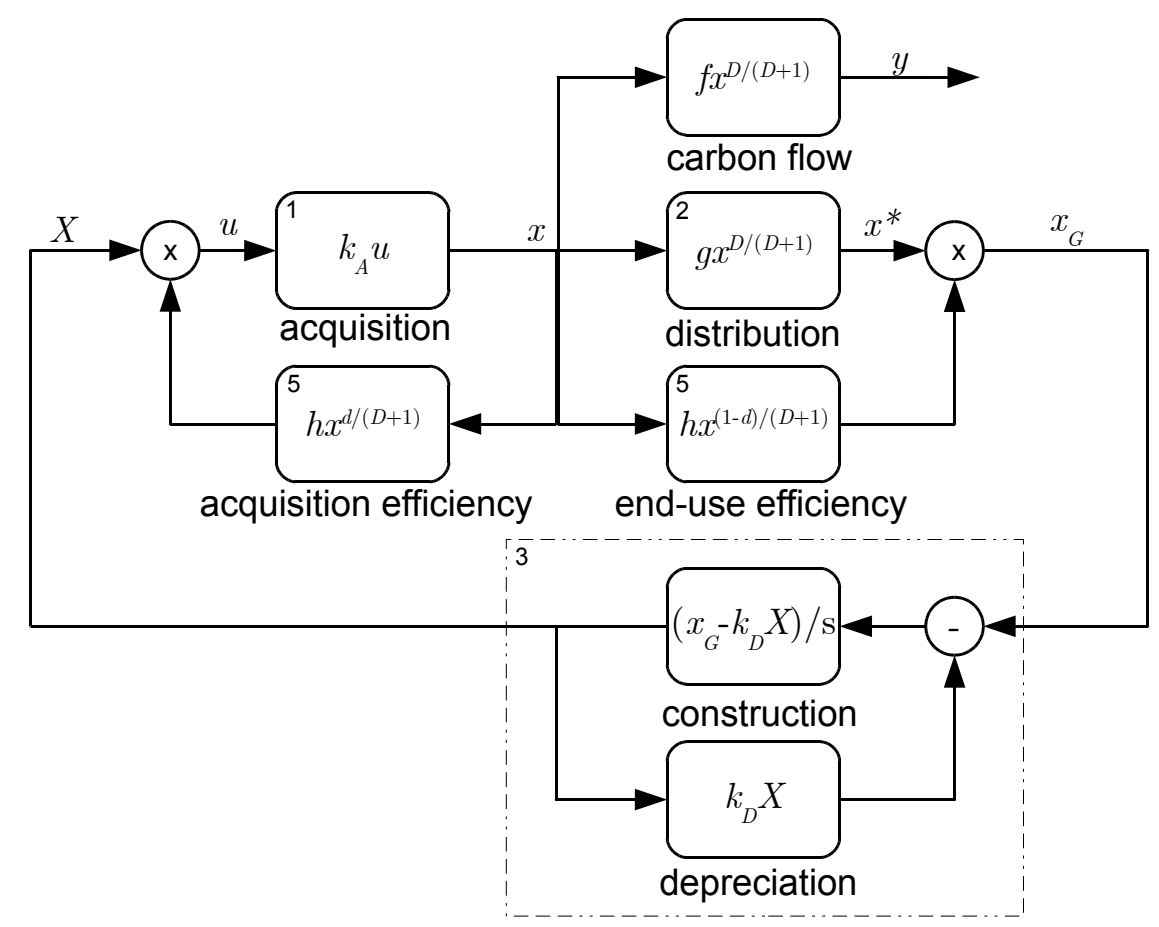

Figure 5. The system diagram representation of the endogenous growth model set out in Eqs. (1)-(5). Numbers in boxes denote which equations apply. $s$ in the "construction" transfer function is the derivative operator, $\mathrm{d} / \mathrm{d} t$.

and end-use efficiencies in the global RADE system. This suggests that industrial society is somehow self-regulated such that the relative growth rates of, for example, energy use, are held near-constant in the long run.

If there is a tendency in industrial society to implicitly regulate growth in things such as energy use, insights into this could be obtained from considering the $\sim 2.4 \% \mathrm{yr}^{-1}$ long-term growth rate on which industrial society appears to settle. At this point, we note that a relative growth rate of $a=2.4 \% \mathrm{yr}^{-1}$ corresponds to a growth timescale of $a^{-1}=42$ years. It would therefore appear sensible to attempt to understand growth in the context of this timescale.

To explore the possible relationships between $a$ and the timescale $a^{-1}$ we start by assuming that the optimisation of the distribution component of the RADE network, combined with the increasing acquisition and end-use efficiencies to control growth (as implied by the control in Eq. 5), point to energy efficiency being an important systemic consideration. Energy efficiency improvements of any kind amount to actions taken to reduce waste and hence increase energy available for specific end uses. Although end-use is notoriously difficult to specify, in the highly reduced description of the global energy system offered above, this end-use can be summarised simply as the work done to expand the size of industrial society. As a result, we refer to the energy not used directly in this work as "supportive" energy use, $x_{\mathrm{S}}$, i.e. energy supporting, but not directly used, in growth. Systemwide optimal energy efficiency improvements imply that $x_{\mathrm{S}}$ is minimised in order to liberate as much energy for growth as necessary. Examples of supportive energy might be the energy expended on exploring, acquiring and distributing resources, personal transport, waste heat and light, etc. Examples of energy directly used for growth, $x_{\mathrm{G}}$, would be energy used to construct, replace and repair the physical components of industrial society such as buildings, oil wells, pipelines, power stations, electricity grids, roads, railways etc.

We can express this supportive energy simply as

$x_{\mathrm{S}}=x-x_{\mathrm{G}}=(1-\varepsilon) x$.

This definition of supportive energy may, at first, appear counter-intuitive because a significant proportion of $x_{\mathrm{S}}$ (such as personal transport) may be thought of as being useful to society. However, in the spatial context considered here, the components of $x_{\mathrm{S}}$ simply represent expenditures of energy necessary to facilitate the useful work of actually expanding the size of industrial society.

If industrial society does indeed attempt to minimise supportive energy use then we should be able to identify a value of $a$ that minimises $x_{\mathrm{S}}$ over a given timescale, $T$. Noting that Eq. (6) resolves to $x=e^{a t}$, and combining within Eq. (8) gives

$X_{\mathrm{S}}=a^{-1}(1-\varepsilon) e^{a T}$,

where $X_{\mathrm{S}}$ is supportive energy accumulated over the integration timescale $T$. We can now differentiate Eq. (9) with 


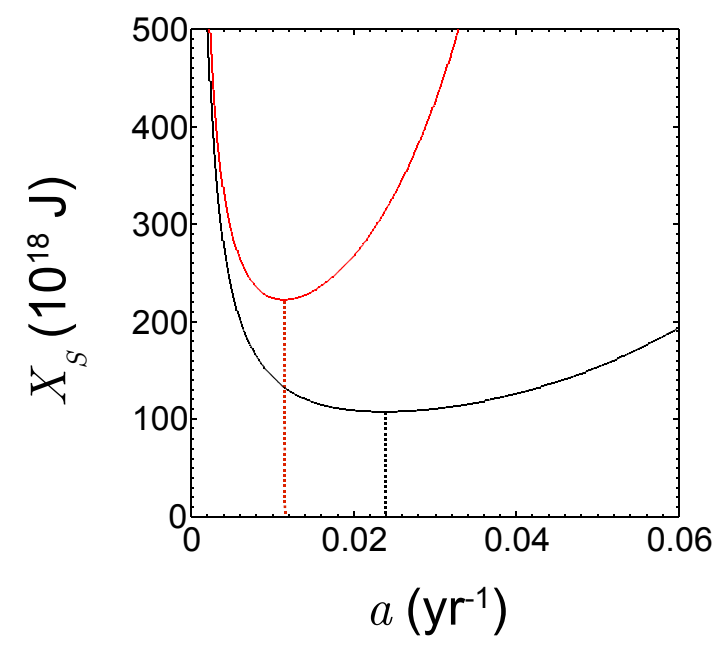

Figure 6. The relationship between the relative growth rate on global primary energy, $a$, and the total energy not directly used in growth, $X_{\mathrm{S}}$. Two scenarios are presented, one with an integration timescale of $T=42$ years (-) and one with an integration timescale of $T=84$ years $(-)$.

respect to $a$ to find the value of $a$ that minimises $X_{\mathrm{S}}$ and, by implication, maximises growth over this timescale. Hence,

$\frac{\mathrm{d} X_{\mathrm{S}}}{\mathrm{d} a}=\frac{(1-\varepsilon) T e^{a T}}{a}-\frac{(1-\varepsilon) e^{a T}}{a^{2}}$

which, for $\mathrm{d} X_{\mathrm{S}} / \mathrm{d} a=0$, has a minimum in $X_{\mathrm{S}}$ at $T=a^{-1}$. Therefore, the growth rate of such a system is fundamentally linked to the timescale over which the system behaviour is optimised with respect to $x_{\mathrm{S}}$.

Figure 6 shows the relationship between $a$ and $X_{\mathrm{S}}$ predicted by Eq. (9). The minimum in $X_{\mathrm{S}}$ with respect to $a$ can be understood in that, for any given integration timescale $T$, if $a$ is below its optimum then the system experiences disproportionate short-term increases in $x_{\mathrm{S}}$ and hence in $X_{\mathrm{S}}$ (Eq. 8). However, if $a$ is above its optimum the system experiences disproportionate long-term increases in $X_{\mathrm{S}}$ because of the effects of enhanced growth (Eq. 6).

Having established a possible connection between the long-run relative growth in global primary energy use, $a \approx 2.4 \% \mathrm{yr}^{-1}$, and the associated timescale, $a^{-1}=42 \mathrm{yr}$, the question remains, why does growth proceed on this timescale? As pointed out above, both technologies in general (Grübler et al., 1999) and large power schemes in particular (Davis et al., 2010) have average lifetimes of $\sim 40$ years. However, as also noted above, these may simply be manifestations of the need to evolve the global energy portfolio in line with its growth rate in order to allow for the required rate of uptake of innovations. Therefore, we look to an alternative explanation of the underlying driver for growth organised at this $\sim 40$ year timescale.

Thus far, we have largely avoided discussing the role of the now 7 billion agents involved in making the decisions that lead to the observed emergent behaviour we have attempted to describe above. We note that where observations are available, $\sim 40$ years is the average working lifetime of people in industrial societies and that this has been a relatively constant property of industrial societies (Ausbel and Grübler, 1995; Conover, 2011) despite the very significant improvements in overall life expectancy in most countries. In addition to the empirical observation that working lifetimes have been stable at around 40 years for a long time, the reason we might implicate working lifetimes as a possible factor on which growth might be organised is that it is only during this timeframe that people can exert influence over the decisions governing the evolution of industrial society. Prior to working, or during retirement, although people are using resources, they are not directly able to influence the evolution of the system. If during their working lifetimes the objective is to seek out near-optimal energy efficiency improvements and hence, by implication, to maximise work done, then this should be sufficient to result in $a \sim T^{-1} \sim 2.4 \% \mathrm{yr}^{-1}$.

Figure 6 also shows that the objective function (Eq. 9) is more sensitive to changes in $a$ below the optimum than above it. If this is true it would explain why periods of below optimum growth are more acutely experienced by industrial societies than are periods of above optimum growth. ${ }^{6}$ Figure 6 also shows the effects of doubling the integration timescale $T$. As $T$ is increased the optimal growth rate falls because the effects of the long-run growth on supportive energy (Eq. 6) weigh more than those of short-term losses (Eq. 8). This is equivalent to an inter-generational view of sustainability in that, by extending the integration interval beyond an individualistic working lifetime, growth is moderated.

\section{Concluding remarks}

In this paper we offer a novel analysis of the behaviour of industrial society based on the physical behaviour of distribution networks. Specifically, we have used global energy use data to explore our hypothesis that industrial society progressively fills space as it grows and that innovations are continually used to overcome the increasing size-related penalties of this growth.

In order for industrial society to grow, the Resource Acquisition, Distribution and End-use (RADE) system must be adaptive because the optimal portfolio of resources and enduses and the appropriate networks linking the two cannot be known a priori. Solving this problem under conditions of relatively deep uncertainty would require forms of dynamic op-

\footnotetext{
${ }^{6}$ In many respects this is linked to the concept of business cycle asymmetries; or what Keynes (1936) referred to as "the phenomenon of the crisis" - the fact that the substitution of a downward for an upward tendency often takes place suddenly and violently, whereas there is, as a rule, no such sharp turning-point when an upward is substituted for a downward tendency.
} 
timisation. As a result, it is not surprising that we see quite rich dynamic behaviour in the growth rate of global primary energy use about its long-run value of $\sim 2.4 \% \mathrm{yr}^{-1}$ (Jarvis and Hewitt, 2014). Such behaviour is clearly not planned centrally, but emerges through the free exchange of information afforded by globalised market mechanisms.

We have identified three distinct points at which we believe the innovations necessary for adaptation occur: at the point of acquisition of resources from the environment; during their distribution; and during their conversion at points of end-use. Without such adaptive capacity both resource availability and their associated distribution costs should limit growth.

Within the framework we have set out, growth in global primary energy use is fundamentally controlled by the optimisation of the RADE system. We have speculated that this optimisation is driven by the inherent desire of people in industrial societies to minimise energy losses and hence maximise work. Since people are only able to significantly influence such decisions during their working lifetimes it may not be surprising that the growth in industrial society appears to be regulated on this timescale.

We acknowledge there are many contentious points in our discussion that challenge conventional views about how industrial society behaves. If it could be stated with confidence that the behaviour of industrial society is largely known, then our attempts to offer an alternative perspective could be considered foolish. However, industrial society must rank as one of the most complex objects in the known universe and our understanding of its behaviour remains poor, to say the least. Utilising theoretical insights from other fields in order to explore this behaviour appears a reasonable strategy. The same can be said for exploiting long-run global energy use data given that changes in energy use are obviously coupled with the evolution of global industrial society. However, significant further work is required to substantiate or refute our arguments. This is ongoing.

Acknowledgements. We thank Piers Forster for suggesting the use of the IEA data and helping define final energy as used in this paper and Bron Szerszynski for valuable discussions. We also would like to acknowledge the now 15 reviewers to date who have offered comments on versions of the manuscript, and in particular Tim Garrett and Mike Raupach. Finally we thank Yan Peng Nie and Stephanie Edeoghon for collating the IEA data. This work was supported by the UK Engineering and Physical Sciences Research Council (EP/I014721/1) and Lancaster University.

The views expressed in this paper are those of the authors and do not necessarily represent the views of, and should not be attributed to, Ofgem or the Gas and Electricity Markets Authority.

Edited by: J. Annan

\section{References}

Ausbel, J.: Regularities in Technological Development: An Environmental View, in: Technology and Environment, edited by: Ausubel, J. H. and Sladovich, H. E., National Academies Press, Washington, D.C., 1989.

Ausbel, J. and Grübler, A.: Working less and living longer: Longterm trends in working time and time budgets, Tech. Forecast. Social Change, 50, 113-131, 1995.

Ayres, R. U.: Energy Inefficiency in the US Economy: A New Case for Conservation. IIASA Research Report RR-89-12, International Institute for Applied Systems Analysis, Laxenburg, 1989.

Banavar, J. R., Moses, M. E., Brown, J. H., Rinaldo, A., Sibly, R. M., and Maritan, A.: A general basis for quarter power scaling in animals, P. Natl. Acad. Sci. USA, 107, 15816-15820, 2010.

Bettencourt, L. M. A.: The origins of scaling in cities, Science, 340, 1438-1441, 2013.

Bettencourt, L. M. A., Lobo, J., Helbing, D., Kühnert, C., and West, G. B.: Growth, innovation, scaling, and the pace of life in cities, P. Natl. Acad. Sci. USA, 104, 7301-7306, 2007.

Boden, T. A., Marland, G., and Andres, R. J.: Global, Regional, and National Fossil-Fuel $\mathrm{CO}_{2}$ Emissions in TRENDS: A Compendium of Data on Global Change, Carbon Dioxide Information Analysis Center, Oak Ridge National Laboratory, US Department of Energy, Oak Ridge, Tenn., USA, doi:10.3334/CDIAC/00001_V2010, 2010.

Conover, C.: The American Health Economy Illustrated, AEI Press, Washington, D.C., 2011.

Dalgaard, C. J. and Strulik, H.: Energy distribution and economic growth, Resour. Energy Econ., 33, 782-797, 2011.

Davis, S. J., Caldeira, K., and Matthews, D. H.: Future $\mathrm{CO}_{2}$ emissions and climate change from existing energy infrastructure, Science, 329, 1330-1333, 2010.

Deng, Y. and Maly, W.: 2.5D System integration: a design driven system implementation schema, in: Proc. Asia South Pacific Design Automation Conf., Yokohama, Japan, 450-455, 2004.

Dittrich, M. and Bringezu, S.: The physical dimension of international trade Part 1: Direct global flows between 1962 and 2005 , Ecol. Econ., 69, 1838-1847, 2010.

ExxonMobil, The Outlook for Energy: a view to 2040, exxonmobil. com/energyoutlook (last access: February 2015), 2013.

Fischer-Kowalski, M and Huttler, W.: Society's Metabolism: The Intellectual History of Materials Flow Analysis, Part II, 19701998, J. Indust. Ecol., 2, 107-136, 1998.

Fouquet, R.: Long run demand for energy services: income and price elasticities over 200 years, Rev. Environ. Econ. Policy, 8, 2-22, 2014.

Garrett, T. J.: Are there basic physical constraints on future anthropogenic emissions of carbon dioxide?, Climatic Change, 104, 437-455, 2011.

Garrett, T. J.: No way out? The double-bind in seeking global prosperity alongside mitigated climate change, Earth Syst. Dynam., 3, 1-17, doi:10.5194/esd-3-1-2012, 2012.

Garrett, T. J.: Long-run evolution of the global economy: 1. Physical basis, Earth's Future, 2, 25, doi:10.1002/2013EF000171, 2014.

Grübler, A.: Technology and Global Change, Cambridge University Press, world primary energy use data downloaded from: http://user.iiasa.ac.at/ gruebler/Data/ 
TechnologyAndGlobalChange/w-energy.csv (last access: October 2015), 2011.

Grübler, A. and Nakienovic, N.: Decarbonising the global energy system, Tech. Forecast. Social Change, 53, 97-110, 1996.

Grübler, A., Nakienovic, N., and Victor, G. B.: Dynamics of energy technologies and global change, Energy Policy, 27, 247280, 1999.

Houghton, R. A.: Carbon Flux to the Atmosphere from Land-Use Changes: 1850-2005 in TRENDS: A Compendium of Data on Global Change, Carbon Dioxide Information Analysis Center, Oak Ridge National Laboratory, US Department of Energy, Oak Ridge, Tenn., USA, doi:10.3334/CDIAC/00001_V2010, 2010.

IEA - International Energy Agency: World Energy Balances, doi:10.5257/iea/web/2012 (last access: 2013), 2012.

Jarvis, A. and Hewitt, C. N.: The "Business-As-Usual" growth of global primary energy use and carbon dioxide emissions - historical trends and near-term forecasts, Earth Syst. Dynam. Discuss., 5, 1143-1158, doi:10.5194/esdd-5-1143-2014, 2014.

Jarvis, A. J., Leedal, D. T., and Hewitt, C. N.: Climate-society feedbacks and the avoidance of dangerous climate change, Nat. Clim. Change, 2, 668-671, 2012.

Keynes, J. M.: The General Theory of Employment, Interest, and Money, Macmillan \& Co. Press, London, 1936.

Macknick, J.: Energy and carbon dioxide emission data uncertainties, IIASA Interim Report IR-09-032, IIASA, Laxenburg, 2009.

Nakicenovic, N.: Decarbonization: Doing More with Less, Technol. Forecast. Social Change, 51, 1-17, 2003.

Nakicenovic, N., Gilli, P. V., and Kurz, R.: Regional and global exergy and energy efficiencies, Energy, 21, 223-237, 1996.

Nelson, R. R. and Wilson, S. G.: An Evolutionary Theory of Economic Change, Harvard University Press, London, 1982.

Nordbeck, S.: Urban allometric growth, Geogr. Ann., 53, 54-67, 1971.
O'Connor, P. A. and Cleveland, C. J.: US Energy Transitions 17802010, Energies, 7, 7955-7993, 2014.

Pauliuka, S., Venkatesha, G., Brattebøa, H., and Müllera, D. B.: Exploring urban mines: pipe length and material stocks in urban water and wastewater networks, Urban Water J., 11, 247-283, 2014.

Peters, G. P., Marland, G., Le Quéré, C., Boden, T., Canadell, J. G., and Raupach, M. R.: Rapid growth in $\mathrm{CO}_{2}$ emissions after the 2008-2009 global financial crisis, Nat. Clim. Change 2, 2-4, 2012.

Rodríguez-Iturbe, I. and Rinaldo, A.: Fractal River Basins: Chance and Self-Organization, Cambridge Univ. Press, New York, 1997.

Savage, V. M., Gillooly, J. F., Woodruff, W. H., West, G. B., Allen, A. P., Enquist, B. J., and Brown, J. H.: The predominance of quarter-power scaling in biology, Funct. Ecol., 18, 257-282, 2004.

Sims, R. E. H., Schock, R. N., Adegbululgbe, A., Fenhann, J., Konstantinaviciute, I., Moomaw, W., Nimir, H. B., Schlamadinger, B., Torres-Martínez, J., Turner, C., Uchiyama, Y., Vuori, S. J. V., Wamukonya, N., and Zhang, X.: Energy supply, in: Climate Change 2007, Mitigation, Contribution of Working Group III to the Fourth Assessment Report of the Intergovernmental Panel on Climate Change, edited by: Metz, B., Davidson, O. R., Bosch, P. R., Dave, R., and Meyer, L. A., Cambridge University Press, Cambridge, 2007.

United Nations: World agriculture: towards 2015/2030, Summary report, Food and Agriculture Organization of the United Nations, Rome, 2002.

West, G. B., Brown, J. H., and Enquist, B. J.: A general model for the origin of allometric scaling laws in biology, Science, 276, 122-126, 1997.

West, G. B., Brown, J. H., and Enquist, B. J.: A general model for ontogenetic growth, Nature, 413, 628-631, 2001. 